\title{
Desperdício de alimentos intra-hospitalar
}

\author{
Food wastage in a hospital
}

Carla Barbosa NONINO-BORGES 1

Estela Iraci RABITO'

Karla da SILVA ${ }^{1}$

Clarice Aparecida FERRAZ2

Paula Garcia CHIARELLO ${ }^{3}$

José Sebastião dos SANTOS ${ }^{4}$

Júlio Sérgio MARCHINI ${ }^{1}$

\section{R E S U M O}

\section{Objetivo}

Verificar a ocorrência do desperdício, na forma de resto de alimentos, na Unidade de Alimentação e Nutrição da Unidade de Emergência do Hospital das Clínicas da Faculdade de Medicina de Ribeirão Preto, da Universidade de São Paulo.

\section{Métodos}

Realizou-se análise descritiva de dados, levantados em um período de 14 dias, sobre os restos de alimentos deixados pelos clientes/pacientes, assim como dos custos estimados do total da produção e do resto de alimentos.

\section{Resultados}

Foram analisadas 650 dietas, totalizando $402 \mathrm{~kg}$ de alimentos, tendo retornado à Unidade de Alimentação e Nutrição $123 \mathrm{~kg}(31 \%)$ de alimentos. Das dietas distribuídas, 71 (15\%) retornaram intactas. Desconsiderando as dietas intactas, o peso total de alimentos produzidos foi de $353 \mathrm{~kg}$ e o resto de $77 \mathrm{~kg}(22 \%)$. O custo total mensal estimado das dietas foi de US $\$ 25,000.00$ e o custo estimado dos restos foi de US $\$ 7,580.00$.

\section{Conclusão}

Os resultados demonstram uma perda excessiva de alimentos, incompatível com as normas operacionais de uma Unidade de Alimentação e Nutrição hospitalar, o que nos remete à necessidade de reflexão acerca das

\footnotetext{
1 Divisão de Nutrologia, Hospital das Clínicas, Faculdade de Medicina de Ribeirão Preto, Universidade de São Paulo. Av. Bandeirantes, 3900, 14049-900, Ribeirão Preto, SP, Brasil. Correspondência para/Correspondence to: C.B. NONINO-BORGES. E-mail: <cnoni@hotmail.com>.

2 Escola de Enfermagem, Faculdade de Medicina de Ribeirão Preto, Universidade de São Paulo. Ribeirão Preto, SP, Brasil.

3 Curso de Nutrição e Metabolismo, Faculdade de Medicina de Ribeirão Preto, Universidade de São Paulo. Ribeirão Preto, SP, Brasil.

${ }^{4}$ Departamento de Cirurgia e Anatomia e Unidade de Emergência, Hospital das Clínicas, Faculdade de Medicina de Ribeirão Preto, Universidade de São Paulo. Ribeirão Preto, SP, Brasil.
} 
possibilidades de ajustes, introduzindo revisões nas práticas gerenciais e operacionais do serviço com enfoque nas ações interdisciplinares.

Termos de indexação: desperdício de alimentos; serviços de alimentação; unidade de alimentação e nutrição hospitalares.

\section{A B S T R A C T}

\section{Objective}

The aim of the current study was to assess food wastage in the Hospital Emergency Section of the Clinical Hospital of the School of Medicine of Ribeirão Preto, University of Sao Paulo and to propose a strategy to face this problem.

\section{Methods}

Food wastage by patients and hospital staff was analyzed. The cost of all the food, supplied and wasted, was estimated.

\section{Results}

The results were calculated based on descriptive analytical data collected during a $14 d$ period. A total of 650 meals were analyzed, totaling $402 \mathrm{~kg}$ of food, from which $123 \mathrm{~kg}(31 \%)$ returned to the Division. From the delivered meals, 71 (15\%) were returned intact, that is, they had not been tried by the patient. Disregarding the returned-intact meals, $353 \mathrm{~kg}$ of food were offered and $77 \mathrm{~kg}(22 \%)$ were wasted. The estimated cost of the total food provision and wastage was US $\$ 25,000$ and US $\$ 7,580.00$ respectively.

\section{Conclusion}

The results reveal large food wastage, unacceptable by the operational rules of a nutrition service in a hospital. Corrective measures, as administrative and operational changes, are necessary. Interdisciplinary actions may be mandatory to reduce food wastage.

Indexing terms: waste of food; food services; hospital food and nutrition services.

\section{N T R O D U Ç Ã O}

Uma alimentação equilibrada é um recurso importante e, muitas vezes, vital para o restabelecimento de enfermos, e a dietoterapia tem papel na recuperação e conservação da saúde ${ }^{1}$. Dentro de um hospital, a unidade de alimentação e nutrição (UAN) é a área de produção de refeições que tem a finalidade de comprar, receber, armazenar e processar alimentos, para posterior distribuição de refeições aos diferentes tipos de clientes/pacientes.

É responsabilidade do nutricionista adequar a oferta dos alimentos à prescrição dietética de cada cliente/paciente, devendo respeitar não somente suas preferências, mas também as limitações impostas pelas doenças, assim como a disponibilidade de pessoal e financeira do serviço².

Na instituição hospitalar, a compatibilidade entre a oferta e as necessidades nutricionais e terapêuticas dos clientes/pacientes, assim como as responsabilidades econômico-administrativas da UAN, são processos complexos, adicionados à programação e execução das refeições. Existem estudos demonstrando a subavaliação do estado nutricional e da capacidade de ingestão alimentar dos clientes/pacientes, resultando em ingestão inadequada de proteínas e calorias, bem como na produção superestimada de alimentos, resultando em desperdício ${ }^{3,4}$.

Há o entendimento de que o equilíbrio entre as necessidades nutricionais dos clientes/pacientes e os custos da dieta pode ser garantido no planejamento dos cardápios, por meio da determinação dos tipos de alimentos e da quantidade a ser adquirida, considerando aspectos como sazonalidade e perenidade, do mesmo modo que as preparações a serem executadas. O controle da produção, da distribuição das refeições, a verificação dos restos e o 
conhecimento das grandes áreas ou variáveis que devam ser melhoradas, por meio de mão de obra e supervisão especializada, podem contribuir para a eficiência da UAN, gerando racionalização com qualidade ${ }^{5,6}$. No gerenciamento de uma UAN, o controle de desperdício é um fator de grande relevância, pois se trata de uma questão não somente ética, mas também econômica e com reflexos políticos e sociais para o profissional nutricionista, tendo em vista que o Brasil é um país onde a fome e a miséria são consideradas como alguns dos problemas de saúde pública ${ }^{7}$.

Dentro do custo mensal de uma UAN estão incluídos os custos da sobra limpa (alimentos produzidos e não distribuídos à clientela) e de resto (alimentos distribuídos e não consumidos pela clientela) $)^{8}$. Isto posto, espera-se que os restos produzidos pelos serviços não ultrapassem 5\% da produção de alimentos, o que os classifica na condição de ótimos. Aqueles serviços cujo desperdício de alimentos varia entre 5\% e 10\% são classificados como bons e na faixa regular estão os serviços que perdem entre 10\% e 15\%. As perdas alimentares que superam 15\% da produção representam um indicativo de péssimo desempenho do serviço 9 .

Apesar da disponibilidade de alimentos para oferta ao cliente/paciente, uma boa parte deles pode ingerir uma quantidade insuficiente para o suprimento de suas necessidades. A ingestão insuficiente é sempre atribuída, primeiramente, aos aspectos clínicos, mas há outras causas não ligadas diretamente à doença e/ou tratamento, por exemplo, as inadequações do serviço de alimentação e nutrição. Algumas situações, como oferta insuficiente de alimentos, rejeição de alimentos por falta de sabor, inexistência de opções de escolha no cardápio, falta de porções menores e energeticamente mais densas para grupos especiais (idosos e crianças) e a pouca conscientização da equipe para a prescrição de dietas muito restritas, apenas em casos estritamente necessários, são algumas das causas geralmente levantadas para a ingestão insuficiente ${ }^{10}$.
Teoricamente, a dieta prescrita ao cliente/paciente seria a mais adequada ao suprimento de suas necessidades nutricionais, porém, em muitos casos, ela parece se apresentar de forma não facilmente aceita pelos clientes/pacientes com apetite diminuído 4 .

O desperdício de alimentos pode ser influenciado por uma série de fatores, como: planejamento inadequado de refeições, preferências alimentares, treinamento dos funcionários para produção e porcionamento dos alimentos ${ }^{8}$. A padronização de porções alimentares dietéticas demanda pesquisa e avaliação individual do cliente/paciente, a fim de garantir as quantidades adequadas $^{11}$ e se espera que isso resulte em melhor qualidade do serviço, além de melhor atenção nutricional.

Considerando-se que o desperdício de alimentos pode ser fator limitante dentro de uma UAN, os objetivos deste trabalho foram verificar sua ocorrência na Unidade de Alimentação e Nutrição da Unidade de Emergência do Hospital das Clínicas da Faculdade de Medicina de Ribeirão Preto, da Universidade de São Paulo (UEHCFMRP-USP), e discutir os pontos mais relevantes que envolvem o problema.

\section{MÉ TOD OS}

A Unidade de Emergência do Hospital das Clínicas da Faculdade de Medicina de Ribeirão Preto é um Hospital com 163 leitos de internação e 46 leitos de observação. Entre os leitos, 22 são destinados a pacientes críticos, 17 a pacientes em cuidados semi-intensivos e 18 a pacientes com doenças infecto-contagiosas. Esta unidade está vinculada, administrativamente, ao Hospital das Clínicas da Faculdade de Medicina de Ribeirão Preto, da Universidade de São Paulo (HCFMRP - USP) e funciona como referência para as urgências e emergências de média e, sobretudo, de alta complexidade para uma região de 4 milhões de habitantes.

A UAN da UEHCFMRP-USP é responsável pela produção de refeições e, atualmente, conta 
com 9 cozinheiras, 48 auxiliares de cozinha e 5 funcionários de armazenagem. O trabalho de acondicionamento e despensa é apoiado por 11 copeiras e 8 auxiliares de nutrição, dividido em 3 turnos. A supervisão de todo o processo é feita por 6 nutricionistas: sendo 2 para produção, 2 para gerenciamento e coordenação e 2 nutricionistas clínicos. A título de ilustração, a média anual de internação é de cerca de 12 mil pacientes; a taxa de ocupação média de $91,0 \%$, a média de permanência de 4 dias, o coeficiente de mortalidade de $6 \%$ e a taxa de infecção hospitalar de $3,3 \%$.

A prescrição da dieta é feita pela equipe médica (médicos residentes com supervisão dos médicos assistentes ou docentes) e repassada, para a produção da UAN, pelos auxiliares de nutrição.

A oferta da dieta aos clientes/pacientes é auxiliada pelos seus acompanhantes e auxiliares de enfermagem, com supervisão dos enfermeiros. Desde o ano 2000, as visitas aos leitos de observação e enfermarias são diárias, das 13 às 21 horas, e é garantida a presença do acompanhante em tempo integral para crianças, adolescentes, idosos e pacientes com necessidades especiais, o que tem ampliado os esforços no incentivo aos pacientes quanto à aceitação alimentar.

Durante um período de 14 dias, foram realizadas, na UEHCFMRP-USP, além das habituais medidas do porcionamento das dietas oferecidas aos clientes/pacientes da UAN, a pesagem dos restos alimentares deixados nas bandejas ou pratos.

O procedimento foi realizado nas enfermarias que abrigam crianças e adultos, das clínicas pediátrica, médica, neurológica e cirúrgica, tendo sido igualmente desenvolvido no refeitório utilizado pelos médicos e servidores da UAN lotados no Hospital. O sistema de distribuição de refeições para pacientes é centralizado e, no refeitório, o sistema utilizado é self-service.

As medidas foram feitas por nutricionistas em dois momentos: na esteira, imediatamente após a montagem das dietas pela UAN, e no retorno, para verificação do respectivo resto alimentar. Os recipientes vazios e os intactos também retornavam à UAN. O mesmo procedimento foi utilizado no cálculo dos restos provenientes do refeitório dos servidores.

Todas as dietas foram oferecidas em pratos rasos de porcelana ou em recipientes de alumínio descartáveis. Para pesagem dos alimentos foi utilizada balança eletrônica marca Filizola ${ }^{\circledR}$, com capacidade para $2 \mathrm{~kg}$ e precisão de $1 \mathrm{~g}$, com estorno dos pesos dos recipientes.

Com base no levantamento do número de refeições servidas mensalmente e no custo da refeição, calculado pelo setor administrativo da instituição, levando em consideração os gastos envolvidos com matéria prima, mão de obra, e material de consumo para preparo e acondicionamento, foi calculado o custo mensal total do almoço e jantar, bem como o custo dos restos alimentares. Os dados apresentados são provenientes de uma análise descritiva dos dados levantados durante 14 dias na UAN da UEHCFMRP-USP.

\section{RE S U L T A D OS}

Das 650 dietas verificadas, 477 (correspondentes a 237 almoços e 240 jantares) foram distribuídas nas diferentes unidades assistenciais da UEHCFMRP-USP, e 173 (119 almoços e 54 jantares) foram destinadas ao refeitório dos servidores (Tabela 1).

Tabela 1. Distribuição dos dias, locais da coleta e número de dietas analisadas para o cálculo do resto alimentar na UEHCFMRP-USP. Ribeirão Preto.

\begin{tabular}{llc}
\hline $\begin{array}{l}\text { Ordem dos dias } \\
\text { da coleta }\end{array}$ & Local (enfermaria) & Dietas $(n=650)$ \\
\hline $1,5,6$ e 7 & Sala de Urgência & 166 \\
10 e 11 & UQ e MI* & 73 \\
12 e 13 & Clínica Cirúrgica e CTI ** & 112 \\
14 e 17 & Clínica Médica e Neurologia & 69 \\
18 e 19 & Pediatria & 57 \\
20 e 21 & Refeitório & 173 \\
\hline
\end{tabular}

*Unidade de Queimados e Moléstias Infecciosas; **Centro de Terapia Intensiva. 
Levando em conta as 650 dietas, foi produzido um total de $402 \mathrm{~kg}$ de alimentos, sendo $220 \mathrm{~kg}$ no almoço e $182 \mathrm{~kg}$ no jantar. Retornou à UAN um total de $123 \mathrm{~kg}$ (33\%) de alimentos, sendo $58 \mathrm{~kg}$ no almoço $(27 \%)$ e $65 \mathrm{~kg}$ no jantar (35\%), que foram considerados restos alimentares.

Das 477 dietas distribuídas nas unidades assistenciais, 71 (15\%) voltaram à UAN intactas. A enfermaria que compreende à Clínica Médica e à Neurologia teve os maiores percentuais de retorno (31\% no almoço e $24 \%$ no jantar). O percentual de retorno foi maior no jantar (17\%) que no almoço (13\%), e se observa, ainda, grande variação entre as diversas clínicas (Tabela 2 ). Desconsiderando-se as dietas intactas e analisando-se apenas as dietas consumidas, a produção de alimentos foi de $353 \mathrm{~kg}$, $(202 \mathrm{~kg}$ no almoço e $151 \mathrm{~kg}$ no jantar). O resto encontrado foi de $77 \mathrm{~kg}$, $40 \mathrm{~kg}$ (20\%) no almoço e $37 \mathrm{~kg}$ (25\%) no jantar.

A Tabela 3 mostra a produção total de alimentos (em kg), separados por refeição (almoço

Tabela 2. Número de dietas intactas que retornaram à Unidade de Alimentação e Nutrição (UAN) da UEHCFMRP-USP, de acordo com as enfermarias. Ribeirão Preto.

\begin{tabular}{|c|c|c|c|c|c|c|c|c|}
\hline \multirow{3}{*}{ Enfermarias } & \multicolumn{2}{|c|}{ Dietas distribuídas (n) } & \multicolumn{6}{|c|}{ Dietas intactas } \\
\hline & \multirow{2}{*}{$\frac{\text { Almoço }}{n}$} & \multirow{2}{*}{$\frac{\text { Jantar }}{\mathrm{n}}$} & \multicolumn{2}{|c|}{ Almoço } & \multicolumn{2}{|c|}{ Jantar } & \multicolumn{2}{|c|}{ Média diária (\%) } \\
\hline & & & $\mathrm{n}$ & $\%$ & $n$ & $\%$ & $\mathrm{n}$ & $\%$ \\
\hline Sala de urgência & 85 & 81 & 7 & 8 & 12 & 15 & 5 & 11 \\
\hline $\mathrm{UQ}+\mathrm{MI}^{*}$ & 32 & 41 & 4 & 13 & 1 & 2 & 3 & 7 \\
\hline Clínica cirúrgica e CTI ** & 59 & 53 & 3 & 5 & 15 & 28 & 9 & 16 \\
\hline Clínica médica e neurológica & 35 & 34 & 11 & 31 & 8 & 24 & 10 & 28 \\
\hline Pediatria & 26 & 31 & 5 & 19 & 5 & 16 & 5 & 18 \\
\hline Total & 237 & 240 & 30 & 13 & 41 & 17 & 36 & 15 \\
\hline
\end{tabular}

* Unidade de queimados e moléstias infecciosas; **Centro de terapia intensiva.

Tabela 3. Quantidade média ( \pm DP) de resto alimentar desconsiderando as dietas íntegras que retornaram à Unidade de Alimentação e Nutrição (UAN), de acordo com a enfermaria da UEHCFMRP-USP. Ribeirão Preto.

\begin{tabular}{|c|c|c|c|c|c|c|c|}
\hline Dietas & $\begin{array}{l}\text { Sala de } \\
\text { urgência }\end{array}$ & UQ/MI * & $\begin{array}{l}\text { Clinica médica } \\
\text { e neurologia }\end{array}$ & Clinica cirúrgica & Pediatria & Refeitório & Total \\
\hline & \multicolumn{7}{|c|}{ Almoço $=326$ dietas } \\
\hline Número de dietas & 78 & 28 & 56 & 24 & 21 & 119 & 326 \\
\hline Produção total (kg) & 48 & 17 & 35 & 15 & 13 & 74 & 201 \\
\hline Resto total $(\mathrm{kg})$ & 8 & 7 & 8 & 5 & 3 & 9 & 40 \\
\hline Resto per capita (kg) & 0,11 & 0,25 & 0,15 & 0,20 & 0,13 & 0,07 & $0,15 \pm 0,07$ \\
\hline \multirow[t]{2}{*}{ Resto $(\%)$} & 17 & 41 & 24 & 32 & 21 & 11 & $26 \pm 11$ \\
\hline & \multicolumn{7}{|c|}{ Jantar $=245$ dietas } \\
\hline Número de dietas & 69 & 40 & 38 & 18 & 26 & 54 & 245 \\
\hline Produção total (kg) & 43 & 25 & 23 & 11 & 16 & 33 & 151 \\
\hline Resto total $(\mathrm{kg})$ & 13 & 5 & 6 & 4 & 2 & 7 & 37 \\
\hline Resto per capita (kg) & 0,18 & 0,12 & 0,17 & 0,21 & 0,09 & 0,13 & $0,15 \pm 0,04$ \\
\hline \multirow[t]{2}{*}{ Resto $(\%)$} & 30 & 20 & 27 & 33 & 15 & 20 & $23 \pm 7$ \\
\hline & \multicolumn{7}{|c|}{ Almoço + Jantar = 571 dietas } \\
\hline Número de dietas & 147 & 68 & 94 & 42 & 47 & 173 & 571 \\
\hline Produção total (kg) & 91 & 42 & 58 & 26 & 29 & 107 & 353 \\
\hline Resto total $(\mathrm{kg})$ & 21 & 12 & 15 & 9 & 5 & 16 & 77 \\
\hline Resto per capita (kg) & 0,14 & 0,18 & 0,16 & 0,20 & 0,11 & 0,09 & $0,15 \pm 0,04$ \\
\hline Resto (\%) & 23 & 29 & 25 & 33 & 18 & 14 & $24 \pm 7$ \\
\hline
\end{tabular}

*Unidade de queimados e moléstias infecciosas. 
Tabela 4. Caracterização do custo total de dietas oferecidas e do desperdício, durante um mês, da Unidade de Alimentação e Nutrição (UAN) da UEHCFMRP - USP. Ribeirão Preto.

\begin{tabular}{lccccc}
\hline & Clientes & Refeitório* $^{*}$ & Total & Custo mensal total $(\text { US } \$)^{\star *}$ Desperdício mensal $(\text { US } \$)^{\star *}$ \\
\hline Almoço & 5376 & 4290 & 9666 & $14,925.44$ & $3,880.61$ \\
Jantar & 4698 & 2856 & 7554 & $9,997.94$ & $3,699.24$ \\
\hline Total & 10074 & 7146 & 17220 & $24,923.38$ & $7,579.85$ \\
\hline
\end{tabular}

*Refeitório do pessoal técnico, dentro da Unidade de Emergência; ** cotação do dia: US $\$ 1.00=R \$ 3,4$.

e jantar), e o resto alimentar conforme as diferentes áreas analisadas, desconsiderando o peso das dietas intactas que retornam à UAN. Verifica-se que há elevado percentual de restos, em média $24 \pm 7 \%$ per capita, com variações significantes entre as diversas unidades, independentemente da refeição. Comparativamente, no refeitório, onde há seleção da quantidade pelo usuário, o percentual de restos foi menor (14\%). A pediatria também apresentou baixo percentual de restos (18\%) em relação às diversas unidades assistenciais.

De acordo com a Tabela 4, foram distribuídas um total de 17220 dietas, considerando-se almoço e jantar, atendendo, tanto os clientes/pacientes quanto o pessoal técnico. Verifica-se também, o custo mensal estimado dessas dietas e dos restos, com base em projeção, a partir dos dados coletados.

\section{I S C U S S Ã O}

Neste trabalho constatou-se que existe, na UAN da UEHCFMRP-USP, uma quantidade excessiva de restos alimentares. Considerando a perda média de 33\% da quantidade total dos alimentos oferecidos, o desempenho efetivo do serviço pode ser classificado como de baixa qualidade ${ }^{9}$. Estes resultados apontam para a necessidade de reorganização dos processos de trabalho do serviço em questão.

Constatamos que as perdas alimentares são mais expressivas nas enfermarias, sobretudo na que agrega a Clínica Médica e a Neurologia, nas quais o número de refeições que retornam intactas atinge $28 \%$. Esta enfermaria concentra os pacientes idosos e com dependência para se alimentarem, com várias comorbidades que são associadas à anorexia ou hiporexia. Todavia, a ineficiência atinge também o refeitório dos servidores, denotando o caráter sistemático do problema.

Foram observadas, durante o estudo, inadequações que podem ser segmentadas em duas categorias: aquelas ligadas à prescrição e outras relacionadas à produção e distribuição das dietas, tais como: a prescrição médica de dieta geral para clientes/pacientes sem dentição e com alterações no nível de consciência; o preparo de dieta para clientes/pacientes que estavam em jejum ou haviam recebido alta; a coleta incorreta da prescrição médica por parte dos auxiliares de nutrição; a falta de ajuste da prescrição dietética à prescrição médica; a falta de padrão durante o porcionamento e a baixa assistência aos clientes/ pacientes que não conseguem se alimentar sozinhos.

Também foram constatados, na UAN, a baixa qualificação da mão de obra existente, por falta de treinamento, motivação e atualização do pessoal; a inadequação e falta de equipamentos e espaço físico e um alto índice de afastamentos por licenças dos servidores. É importante ressaltar que, em uma Unidade de Alimentação e Nutrição, os funcionários são responsáveis pela prestação de serviços e devem estar comprometidos com as metas institucionais?.

De acordo com a resolução do CFN 223/99, do Conselho Federal de Nutricionistas, é de competência do nutricionista avaliar a dieta, por meio de diferentes métodos, diagnosticando sua adequação frente às necessidades nutricionais 
e dietoterápicas, considerando os hábitos alimentares, incluindo padrão alimentar quanto ao número, tipo e composição das refeições, rotinas, restrições, preferências alimentares e apetite ${ }^{2}$. Na prática, o que acontece nesta instituição e, em muitos outros serviços hospitalares, é o não atendimento ao previsto pela Resolução n 63/2000 da Agência Nacional de Vigilância Sanitária (Anvisa), segundo a qual a prescrição dietética deve ser feita por um nutricionista, inserido na equipe multiprofissional envolvida na responsabilidade do atendimento ao cliente/paciente ${ }^{12}$. A disponibilidade de nutricionistas com treinamento e competência clínica para se responsabilizarem pela prescrição dietética, de modo a atender as necessidades do cliente/paciente, como parte geral do tratamento proposto, é condição fundamental para o bom funcionamento de um serviço de alimentação e nutrição.

Os ajustes da prescrição da dieta, por meio do aperfeiçoamento da anamnese médica e nutricional, com inclusão das observações do enfermeiro e outros profissionais acerca do apetite e do padrão de ingestão, poderiam diminuir o desperdício em decorrência da aproximação da dieta às necessidades e à preferência dos clientes/ pacientes ${ }^{13}$. No entanto, as responsabilidades desses profissionais, perante a assistência nutricional, na UEHCFMRP-USP, são representadas pela justaposição dos cuidados formulados pelas respectivas categorias, e distantes de ser um produto da cooperação interdisciplinar, centrada nas necessidades do usuário. Existe uma fragmentação do trabalho dentro da instituição, uma vez que a UAN consignou que, entre as suas próprias atribuições estavam apenas a produção de alimentos com qualidade e a elaboração de dietas, de acordo com a prescrição médica, enquanto os problemas de execução e distribuição deveriam recair sobre a enfermagem.

Fica evidente que a orientação da prática gerencial e assistencial da UAN está centrada na prescrição médica da dieta, e não na prescrição dietética, como seria o ideal. Ademais, o contexto é desfavorável ao desenvolvimento da assistência nutricional a partir da abordagem interdisciplinar, visto que o número de nutricionistas, na maioria dos hospitais, ainda é inferior às necessidades. Um incremento de nutricionistas, capacitados para a prática clínica, participando ativamente e desenvolvendo seu papel junto à equipe multiprofissional, incluindo a avaliação nutricional e a anamnese alimentar, são medidas que podem minimizar este problema.

Além disso, abordagens que procurem atender às expectativas do cliente/paciente, em busca de sua satisfação, são essenciais. Informações sobre o serviço de alimentação para toda a equipe de atendimento, e mesmo para o paciente, diminuiriam a distância entre o mesmo e o cuidado nutricional. A capacitação da equipe para a prescrição de dietas e a implantação de uma padronização das mesmas seriam úteis para minimizar erros e otimizar as prescrições ${ }^{14}$.

No atual contexto gerencial e operacional da produção de refeições, dificilmente serão executados os serviços necessários para o bom funcionamento da UAN. Tampouco, a capacitação dos profissionais da UAN, como estratégia isolada, causará impacto ${ }^{3}$. A fixação de nutricionistas nas distintas unidades assistenciais, certamente, fortalecerá a ação interdisciplinar e, conseqüentemente, a revisão da assistência nutricional. Reportamo-nos ao conceito de produtividade, segundo o qual os processos hospitalares estão sendo revistos, na tentativa de se adequar à melhor forma de rendimento, garantindo efetividade e eficiência. Na Tabela 4 analisamos que os recursos financeiros investidos e, posteriormente, descartados, na forma de restos de alimentos, impõem restrições ao aumento da produtividade hospitalar.

A resolução dos problemas apontados depende do esforço coletivo das unidades gerenciais da instituição, a partir de diagnósticos embasados, como, por exemplo, os dados aqui apresentados. Devido à magnitude do problema, sugerimos, para o seu enfrentamento, a otimização da gestão dos insumos, o aprimoramento no preparo e distribuição das dietas, a capacitação e adequação de recursos humanos e a introdução de mecanismos de coordenação sincronizada entre os produtos ofertados pela UAN e as áreas de 
consumo. Destacamos que, nas unidades assistenciais, o cliente/paciente, o nutricionista, o médico e os profissionais da enfermagem são parte de um círculo de produtividade e qualidade dos produtos dietoterápicos.

No que se refere à prescrição dietética, esta deveria ser definida por um nutricionista, minimizando erros em estimativas de porcionamento, fracionamento de refeições, seleção de alimentos e individualização da dieta.

Perseguindo a eficiência em todos os níveis da assistência nutricional, a equipe de atendimento ao cliente/paciente deveria ser estimulada a trabalhar efetivamente, concretizando a interdisciplinaridade. Isso significa a tomada de consciência, por parte dos profissionais envolvidos, da importância do trabalho de cada categoria, em prol do melhor tratamento do paciente. É obvio que este objetivo só seria atingido com um número adequado de profissionais em cada unidade, possibilitando a prescrição da dieta e a revisão do cuidado nutricional.

Em conclusão, os resultados apontam uma perda excessiva de alimentos, incompatível com as normas operacionais de um serviço de alimentação hospitalar. Frente a estes resultados, fazem-se necessárias medidas corretivas, como revisão das práticas gerenciais e operacionais deste serviço, com enfoque nas ações interdisciplinares.

\section{RE FER Ê NCIAS}

1. Mezomo IB. O serviço de alimentação. In: Mezomo IB. Os serviços de alimentação planejamento e administração. Barueri: Manole; 2002. p.81-273.

2. Brasil Conselho Federal de Nutricionistas. Resolução n²23/1999 de 13 de jul 1999. Dispõe sobre o exercício profissional do nutricionista na área de nutrição clínica e dá outras providências. Diário Oficial da União [Internet]. 1999 set. Disponível em: http://www.cfn.org.br/legislação/ resolução/res223.htm
3. Almdal T, Viggers L, Beck AM, Jensen K. Food production and wastage in relation to nutritional intake in a general district hospital-wastage is not reduced by training the staff. Clin Nutr. 2003; 22(1):47-51.

4. Barton $A D$, Beigg $C L$, MacDonald IA, Allison SP. High food wastage and low nutritional intakes in hospital patients. Clin Nutr. 2000; 19(6):445-9.

5. Giaconi GJ. Algunos aspectos de la productividad em salud. Cuad Med Soc. 1995; 36(1):19-23.

6. Proença RPC, Souza AA, Veiros MB, Hering B. Gestão de cardápios na produção de refeições. In: Proença RPC, Souza AA, Veiros MB, Hering B. Qualidade nutricional e sensorial na produção de refeições. Florianópolis: Editora da UFSC; 2005. p. 55-122.

7. Pereira GS, Castro IR. Considerações sobre o plano de combate à fome e à miséria. Cad Saúde Pública. 1993; 9(Supl 1):106-13.

8. Hirschbruch MD. Unidades de alimentação e nutrição: desperdício de alimentos qualidade da produção. Rev Hig Alim. 1998; 12(55):12-4.

9. Castro FAF, Queiroz VMV. Cardápios: planejamento, elaboração e etiqueta. Viçosa: Universidade Federal de Viçosa; 1998. p.1-29.

10. Dupertuis YM, Kossovsky MP, Kyle WUG, Raguso CA, Genton L, Pichard C. Food intake in 1707 hospitalized patients: a prospective comprehensive hospital survey. Clin Nutr. 2003; 22(2): 115-23.

11. McWhirter JP, Pennington CR. Incidence and recognition of malnutrition in hospital. BMJ. 1994; 308(6934):945-8.

12. Brasil. Agência Nacional de Vigilância Sanitária. Resolução n 63 de 6 de julho de 2000. Diário Oficial da União [Internet]. 1999 jul. Disponível em: http://www.anvisa.gov.br /legis/resol/2000/ 63_00rdc.htm

13. Gall MJ, Grimble GK, Reeve NJ, Thomas SJ. Effect of providing fortified meals and between-meal snacks on energy and protein intake of hospital patients. Clin Nutr. 1998; 17(6):259-64.

14. Watters CA, Sorensen J, Fiala A, Wismer W. Exploring patient satisfaction with foodservice through focus groups and meal rounds. J Am Dietetic Assoc. 2003; 103(10):1347-49.

Recebido em: 10/3/2005

Versão final reapresentada em: 29/9/2005 Aprovado em: 26/10/2005 